
allemande

\title{
Plaidoyer pour le catastrophisme
}

Valentine Erné-Heintz et Brice Martin

\section{(2) OpenEdition}

\section{Journals}

Édition électronique

URL : https://journals.openedition.org/allemagne/2228

DOI : 10.4000/allemagne.2228

ISSN : 2605-7913

Éditeur

Société d'études allemandes

\section{Édition imprimée}

Date de publication : 1 juillet 2020

Pagination : $67-80$

ISSN : 0035-0974

\section{Référence électronique}

Valentine Erné-Heintz et Brice Martin, «Plaidoyer pour le catastrophisme », Revue d'Allemagne et des pays de langue allemande [En ligne], 52-1 | 2020, mis en ligne le 06 juillet 2021, consulté le 19 février 2022. URL : http://journals.openedition.org/allemagne/2228 ; DOI : https://doi.org/10.4000/ allemagne.2228 


\section{Plaidoyer pour le catastrophisme}

\section{- Valentine Erné-Heintz * et Brice Martin **}

\section{Introduction}

Le catastrophisme peut-il être éclairé(1) ? Assurément pour Jean-Pierre Dupuy qui affirme la nécessité de se concentrer sur le caractère inéluctable de la catastrophe pour mieux l'anticiper et pour qui savoir ne suffit pas pour croire:

«On peut se fixer sur le scénario du pire, non pas comme pouvant ou devant se produire dans l'avenir, mais en tant qu'il pourrait ou devrait se produire si l'on entreprenait telle action. Dans le premier cas, le scénario du pire est de l'ordre d'une prévision; dans le second cas, c'est une hypothèse conditionnelle dans une délibération qui doit aboutir à choisir, parmi toutes les options ouvertes, celle ou celles qui rendent ce pire acceptable; ou, dans une autre variante, l'option qui rend ce pire le moins dommageable possible. [...] La catastrophe a ceci de terrible que non seulement on ne croit pas qu'elle va se produire alors même qu'on a toutes les raisons de savoir qu'elle va se produire, mais qu'une fois qu'elle s'est produite elle apparaît comme relevant de l'ordre normal des choses. Sa réalité la rend même banale. Elle n’était pas jugée possible avant qu'elle ne se produise ${ }^{(2)}$.

Autrement dit, le catastrophisme pose la question des aléas extrêmes en même temps que des événements inconnus ou inconcevables. Mais qu'appelle-t-on catastrophisme? L'apocalypse peut-elle être joyeuse ${ }^{(3)}$ ? S'agit-il d'une vision apocalyptique de militants

* Maître de conférences en sciences économiques, Université de Haute-Alsace, CERDACC EA 3992, Mulhouse.

** Maître de conférences en géographie, Université de Haute-Alsace, Directeur du GIS Risques de I'Université de Haute-Alsace depuis 2010.

1 Jean-Pierre Dupuy, Pour un catastrophisme éclairé. Quand l’impossible est certain, Paris, Seuil (La couleur des idées), 2002.

2 Ibid.

3 François JARRIGE, «Jean-Baptiste Fressoz, L'apocalypse joyeuse. Une histoire du risque technologique, collection L'Univers historique, Paris, Seuil, 2012, 320 p. ISBN : 978-2-02-105698-3. 23 euros », Revue d'histoire du XIX ${ }^{e}$ siècle, 45/2 (2012), p. 187-189. 
opposés à la mythologie techniciste ${ }^{(4)}$, d'une perspective présentée par des «collapsologues pessimistes ${ }^{(5)}$ ou d'une réalité que l'on ne veut pas voir et qui paralyse? En fait, l'idée que le pire est possible invite à agir. Toutefois, le contenu de cette action dépend explicitement des connaissances disponibles, donc de la capacité à questionner et à disposer d'outils adéquats ${ }^{(6)}$. Penser la catastrophe réinsère la prudence dans l'analyse du risque en rappelant que la précaution n'est qu'une traduction de l'éthique de responsabilité chère à Hans Jonas ${ }^{(7)}$, une «éthique du futur » construite spécifiquement autour de la menace nucléaire. Elle réaffirme la distinction entre risque avéré et risque potentiel: si le risque avéré ne peut pas être nul, ce n'est pas le cas d'un risque potentiel dont la probabilité d'une occurrence (un scénario ${ }^{(8)}$, une corrélation) est incertaine ${ }^{(9)}$. Autrement dit, le catastrophisme concerne tout autant un risque avéré dont la probabilité est très faible et la gravité forte (séisme majeur, terrorisme, catastrophe nucléaire, crue extrême) qu'un risque potentiel dont le scénario reste à construire faute d'expérience ou de capacité à concevoir l'événement dans ses causes, ses caractéristiques et ses conséquences. Cela se rapporte aux phénomènes rares, inconnus ou oubliés ou aux risques dont les conséquences s'inscrivent dans le temps long et qu'on peine à appréhender à l'heure actuelle (phtalates, perturbateurs endocriniens, effets transgénérationnels). Autrement dit, si nos sociétés sont devenues des «sociétés du risque "(10) en mettant certains risques "sous contrôle» et en devenant résilientes, elles ont aussi minimisé d'autres menaces ou rendu certains risques invisibles ${ }^{(11)}$. La réflexivité ne suffit pas face à certains défis. L'éventualité d'une catastrophe interroge ce cadre normatif de l'analyse du risque et invite à une approche plus globale.

Cette contribution revient sur des situations de rupture ou d'aveuglement statistiquement qualifiées d'événements aberrants qui mettent à l'épreuve la science normale ${ }^{(12)}$, la toxicologie ou les procédures classiques d'expertise ${ }^{(13)}$ au même titre que

4 Comme Georges Bernanos, Ivan Illich, Jacques Ellul, Richard Bartlett Gregg... En réalité, dès les années 30-40, l'École de Francfort (Max Horkheimer, Theodor Adorno, Herbert Marcuse, Walter Benjamin, Jürgen Habermas) souligne le paradoxe du progrès qui devient contre-productif.

5 Laurent AlexAndre, «Stop aux écologistes apocalyptiques», Le Figaro, 7 septembre 2018, p. 16.

6 Valentine Erné-Heintz et Jean-Christophe Vergnaud, «Au sujet du besoin d'un niveau de preuve robuste pour évaluer le risque», Revue Vertigo, 2016, journals.openedition.org/vertigo/17828.

7 Hans Jonas, Le principe responsabilité, une éthique pour la civilisation technologique (Das Prinzip Verantwortung, 1979, trad. de Jean Greisch), Paris, Flammarion, 1988.

8 Citons la question des effets transgénérationnels dans le cas du distilbène ou encore les effets reproductifs des perturbateurs endocriniens: la discussion porte aussi sur l'approche adoptée et l'un des points de divergence se trouve dans la question de la monotonie de la relation dose-réponse.

9 Cette probabilité peut être nulle ou très élevée. Dans le cas d’un risque avéré, la probabilité porte sur la réalisation d'un accident.

10 Ulrich BEck, La société du risque. Sur la voie d'une autre modernité (1986, trad. de l'allemand par L. Bernardi), Paris, Aubier-Alto, 2001.

11 Valentine Erné-Heintz, «Le côté obscur du risque: son invisibilité!», Risques, Études et Observations, $\mathrm{n}^{\circ} 2$ (2017), p. 21-39, www.riseo.cerdacc.uha.fr/, fr.calameo.com/read/005049066fa9bf1a01289.

12 Dominique Bourg, Pierre-Benoît Joly, Alain Kaufmann, Du risque à la menace. Penser la catastrophe, Paris, Presses universitaires de France. 2013.

13 Valentine Erné-Heintz et Denis BARD, «Un expert précautionneux», in: Jean-Paul Markus et Karine Favro (dir.), L'expert dans tous ses états, Paris, Dalloz (coll. Thèmes et commentaires), 2016, p. 197-215. 
nos processus cognitifs. Sont-elles pour autant toujours non prévisibles? Aussi, les propos s'organisent en deux points: premièrement, nous définissons le cadre horsnorme dans lequel cet improbable cygne noir ${ }^{(14)}$ s'épanouit. Deuxièmement, nous caractérisons la notion de catastrophe en insistant sur la nécessité de penser les scénarios catastrophiques pour une meilleure gestion du présent.

\section{Penser l'impossible «cygne noir »...}

Dominique Bourg, Pierre-Benoît Joly et Alain Kaufmann ${ }^{(15)}$ s'interrogent sur la notion de risque elle-même: l'approche classique a objectivé les dangers auxquels nos sociétés sont confrontées, mais elle rend également invisibles ou minimise certaines menaces. L'évaluation au travers des seuls risques mesurables dénature la notion de risque au profit d'une logique administrative et/ou assurantielle: les outils déployés (protections physiques, réparations financières, etc.) font oublier que le pire scénario reste possible voire restreignent le risque naturel à sa seule dimension économique ${ }^{(16)}$. Toutefois, ils contribuent à une certaine désinhibition du fait de cette quantification ou mise en normes de certains risques. La fabrique de la norme qui en découle a incontestablement quelque chose de rassurant sur le plan cognitif: la persuasion de pouvoir maîtriser la nature et/ou la technique.

\section{Des limites de la prospective prévisionnelle dans une situation hors-norme}

Il y a deux manières de se projeter dans un risque. La première façon fait appel à la prospective prévisionnelle: la gestion - et de façon générale la gouvernance - est guidée par l'anticipation de la réalisation d'un risque, potentiellement susceptible de générer une catastrophe. Cette démarche se base sur la description de futurs possibles (des scénarios récapitulant les grandes tendances) en tenant compte de données à la fois quantitatives et qualitatives. Il en est ainsi, par exemple, pour le changement climatique: d'un côté, diminution du nombre de jours de gel, hausse des précipitations ou des jours de sécheresse et de l'autre côté, effets sur la santé des populations vulnérables, sur les cycles végétatifs des cultures (dates de floraison, de débourrement, de véraison) ou les habitudes migratoires de certaines espèces. La prospective anticipe le climat du futur à travers un cumul de précipitations, de vagues de chaleur et de variations des températures avec une répartition saisonnière de leur intensité. Ce scénario étudie les qualités intrinsèques d'un territoire pour imaginer comment celui-ci réagira avec le climat de demain.

14 Nassim Nicholas Taleb, Le cygne noir. La puissance de l’imprévisible, Paris, Les Belles Lettres, 2008.

15 Bourg/Joly/Kaufmann, Du risque à la menace (note 12).

16 La recherche d'une certaine efficacité agronomique, qui agit sur l'aléa par des protections physiques ou des intrants chimiques par exemple, a accru la vulnérabilité des agriculteurs. Vulnérabilité économique tout d'abord car il faut rentabiliser l'installation des mesures de lutte contre l'aléa, mais aussi l'apparition d'une dépendance à ces outils. Pour le géographe Freddy Vinet, «cette mutation sociale de fond n'est pas sans conséquence sur la perception des risques naturels». F. VINET, «La question du risque climatique en agriculture: le cas de la grêle en France», Annales de Géographie, 111/627-628 (2002), p. 592-613. 
Figure 1. Un futur construit

\begin{tabular}{|c|c|}
\hline $\begin{array}{l}\text { Représentation de la situation actuelle: DI } \\
\text { (sensibilité du milieu, type d'aléa, }\end{array}$ & DE L'EXISTANT \\
\hline $\begin{array}{l}\text { HYPOTHĖSES DU PARADIGME } \\
\text { (intensité de l'aléa, perturbations possibles, } \\
\text { exposition future, évolution des habitudes) }\end{array}$ & $\begin{array}{r}\text { FUTUR IMAGINÉ : Scenarii possibles } \\
\text { (identifier les variables clés } \\
\text { d'adaptation et/ou } \\
\text { les mesures d'atténuation) }\end{array}$ \\
\hline
\end{tabular}

Lorsque des scénarios sont affectés de probabilités, cela signifie que l'on attribue à chaque scénario un certain degré de croyance. Cette quantification des scénarios répond souvent à un besoin de clarté pour rationaliser une décision. Mais il est parfois difficile de déterminer - suggérer - des probabilités fiables dans des situations d'incertitude ou d'ambiguïtés scientifiques ${ }^{(17)}$ où le futur reste à imaginer ${ }^{(18)}$. En l'espèce, la possibilité qu'il puisse y avoir des méga-feux dans des régions à hauts revenus comme en Californie ou en Australie en 2019 dépasse l'imagination. Le caractère exceptionnel de ce type de situation spectaculaire ne devient un futur possible qu'une fois réalisée.

En somme, l'objectif n'est pas de prévoir (c'est en cela que la prospective diffère de la prévision) mais de proposer plusieurs visions du monde (états futurs) en intégrant éventuellement des trajectoires de rupture technologique ou climatique par exemple. En matière de santé environnementale, l'épidémiologie et la toxicologie sont confrontées aux limites du principe de Paracelse ${ }^{(19)}$ en raison d'effets des faibles doses et du temps de latence entre l'exposition à une substance et l'observation d'un effet (effet critique adverse, mélange de substances, effets paradoxaux et de bioaccumulation ou encore effet fenêtre). Pour les catastrophes écologiques contemporaines, c'est la possibilité de l'épuisement de certaines ressources (halieutiques, fossiles) ou le risque de non-linéarité des trajectoires technologiques par le biais de l'histoire du capitalisme industriel qui est questionnée ${ }^{(20)}$. Elles ne peuvent être lues comme monolithes; elles ne sont ni mono-causales ni solvables par un simple accord sur le climat. Par ailleurs, elles font apparaître des inégalités environnementales ${ }^{(21)}$ qui se conjuguent avec des inégalités territoriales et socio-économiques. Toute la difficulté est justement d'intégrer de tels schémas catastrophiques qui se transforment alors en véritables défis pour la gouvernance du risque en panne de légitimité.

C'est pourquoi, la seconde façon de se projeter dans les états futurs sollicite le «hors norme», l'impossible. Ici, le risque ne se définit pas comme un ensemble de données à interpréter mais comme une réserve de possibles à imaginer du fait del'intensité extrême de l'aléa et de l'ampleur des enjeux qui rendent le risque incontrôlable. C’est également dans

17 Valentine Erné-Heintz, «Qu'est-ce que la prudence? Le cas du nucléaire », Le journal des accidents et des catastrophes, $\mathrm{n}^{\circ} 87$ (2008), www.jac.cerdacc.uha.fr/.

18 Valentine ERnÉ-Heintz, «Incertitude radicale et choix économique: illustration avec le risque climatique », in: Karine Favro (dir.), L'expertise: enjeux et pratiques, Paris, Lavoisier (coll. Science du risque et du danger), 2009, p. 81-96.

19 Il énonce que «toutes les choses sont poison et rien n’est sans poison; seule la dose détermine ce qui n'est pas poison». Il est à l'origine du principe des seuils et des doses journalières admissibles. Il est adapté à une logique monoproduit avec des effets linéaires.

20 Jean-Baptiste Fressoz et Fabien Locher, «L'agir humain sur le climat et la naissance de la climatologie historique, XVII ${ }^{\mathrm{e}}$-XVIII ${ }^{\mathrm{e}}$ siècles », Revue d'histoire moderne \& contemporaine, 62/1 (2015), p. 48-78.

21 Valentine Erné-Heintz, «Risque et inégalités environnementales: un autre regard sur le risque», Risques, Études et Observations, 2020, www.riseo.cerdacc.uha.fr/ (à paraître). 
cet esprit que Hans Jonas évoque «l'heuristique de la peur» ${ }^{(22)}$ : l'homme est aujourd'hui au cour d'une dynamique irréversible dont les effets cumulatifs à long terme sont autant imprévisibles qu'incontrôlables au point de menacer son existence même, car son savoir prévisionnel demeure en deçà du pouvoir destructeur de la technique. C'est pourquoi il défend une éthique tournée vers l'avenir de la civilisation technologique et basée sur le principe de la responsabilité. L'alarmisme peut alors servir d'aiguillon. La peur est au cœur de cette éthique de la responsabilité: elle pousse à agir car c'est en imaginant le pire qu'il est possible de gérer le quotidien. C'est dans ce sens que se posent certains scénarios apocalyptiques de la NASA sur l'éventualité de départs de feux concomitants à plusieurs endroits de la planète et qui se rejoindraient en provoquant des méga-feux inextinguibles. Cependant, cette façon de penser le risque a des inconvénients. Elle se situe, par définition, dans une approche pessimiste, catastrophiste qui envisage de nouvelles contraintes avec parfois une mise en cause radicale des modes de vie contemporains ou des méthodes d'analyse et d'expertise ${ }^{(23)}$ d'un risque. À ce titre, Ulrich Beck s'intéresse particulièrement aux risques technologiques majeurs qui échappent au contrôle de l'individu: "C'en est assez de la légende de l'imprévisibilité des effets secondaires: les conséquences ne sont pas apportées par les cigognes, on les a fabriquées " ${ }^{(24)}$. D’ailleurs, le développement des questionnements autour de l'anthropocène ${ }^{(25)}$, dès la fin des années 70 , pose un problème inédit: l'observation du risque et la place de l'homme dans ce risque, en tant que victime et cause résiduelle du risque. Ces questions sont concomitantes à l'éclosion d'approches plus qualitatives ${ }^{(26)}$ qui prennent en compte les limites du progrès (pollution industrielle, maladies professionnelles, liens santé/ environnement, pesticides, stress et suicides au travail). Néanmoins, les logiques d'adaptation ou d'atténuation sont souvent défaillantes face à ces nouveaux risques ${ }^{(27)}$. Pour le sociologue Michel Callon:

«Les technosciences produisent des affaires embrouillées. [...] On ne peut plus s'appuyer sur ce que l'on sait pour décider ce que l'on veut, puisque les choix à faire portent prioritairement sur l'identification des problèmes à résoudre et sur ce que l'on souhaite savoir » ${ }^{(28)}$.

Incontestablement, l'outil rend parfois aveugle ${ }^{(29)}$.

Nonobstant, la peur de la catastrophe peut conduire soit à une forme de nihilisme, soit à la négation du risque. Bernadette de Vanssay soulève ainsi le caractère ébranlant d'un aléa exceptionnel à travers une crue exceptionnelle «qui domine la représentation

22 H. JonAs, Le principe responsabilité (note 7).

23 Erné-Heintz/Vergnaud, «Au sujet du besoin d'un niveau de preuve robuste pour évaluer le risque» (note 6).

$24 \mathrm{U}$. Beck, La société du risque (note 10).

25 Certains auteurs préfèrent utiliser le terme de «fracture métabolique»; voir Franck BouTARIC, «L'appropriation de la méthode de l'évaluation des risques sanitaires en France», Écologie \& politique, 40/2 (2010), p. 117-135.

26 Valentine Erné-Heintz, «Les sciences sociales dans l'expertise: entre décomposition et recomposition », Droit de l'environnement, décembre 2014, p. 42-49.

27 Le défi porte à la fois sur la mesure des polluants, sur l'identification et les éventuelles interactions. Ce n'est pas tant une question de toxicité aiguë que d'effets à long terme de très faibles doses.

28 Michel Callon, "Quel espace public pour la démocratie technique?», Les sens du public, PUF, 2003, p. 201, www.u-picardie.fr/curapp-revues/root/48/4._QUEL_ESPACE_PUBLI.pdf_52cfbbffa215a/4._ QUEL_ESPACE_PUBLI.pdf. 
globale de l'inondation" ${ }^{(30)}$. Le neuropsychologue Francis Eustache ${ }^{(31)}$ souligne le rôle des émotions dans la mémoire collective: la chute de la flèche de Notre-Dame de Paris évoque l'image des tours du World Trade Center. Cette déstructuration de l'événement normal par l'éventualité d'une catastrophe ébranle le cadre cognitif du fait d'une nonmaîtrise de son destin (individuel ou collectif) où l'homme ne serait que spectateur de la fin d'un monde. De façon similaire, les théories de l'effondrement ont ouvert un nouveau champ de recherche intitulé la collapsologie. Ces archétypes deviennent des symboles partagés qui résonnent avec intensité: le passé est encore présent et la fin est prévisible. Ils sont irrigués par des mythes, des œuvres artistiques et forment une mémoire collective. Il suffit de se référer à toute une série d'œuvres d'anticipation ou de fictions dystopiques aussi diverses que La Jungle de Upton Sinclair (1904), Make Room! de Harry Harrison (1966) qui inspira Soleil vert de Richard Fleischer (1973), La machine à explorer le temps de H.G. Wells (1895), 2001, L'Odyssée de l'espace de Stanley Kubrick (1968), Mad Max de G. Miller (1979) ou Le dernier homme de Margaret Atwood (2003) pour comprendre comment le catastrophisme peut se décliner. La peur de l'apocalypse est un thème récurrent qui se construit autour de la technologie, de la démographie, de la panique alimentaire ${ }^{(32)}$ ou encore de l'angoisse climatique. Fred Vargas et Greta Thunberg deviennent les nouvelles héroïnes de cet alarmisme écologique.

\section{Laléa exceptionnel: comment ne rien voir conduit à la catastrophe}

Faut-il prévoir le pire pour gérer le quotidien? Où placer l'improbable cygne noir dans le schéma d'analyse du risque? Le plus difficile est d'imaginer un autre cadre d'analyse sans attendre d'être témoin d'une catastrophe, car il sera alors trop tard. La catastrophe exige une autre règle pour anticiper et empêcher le risque redouté qui se réfère à une intensité anormale de l'aléa et/ou à la définition sociale de ce qu'est une catastrophe. Il s'agit bien du caractère «anormal» voire inattendu qu'il convient d'interroger:

«La catastrophe improbable ne s’intéresse pas au risque gérable mais, au contraire, suggère que la survie de l'homme peut être menacée, que l'homme soit aliéné par la technique. Cette question de l'apocalypse surgit très rapidement en raison de la possibilité de dommages irréversibles et de catastrophes redoutables: l'homme est capable de s'autodétruire et de détruire son environnement ${ }^{(33)}$.

Nassim Taleb explique dans sa théorie du cygne noir ${ }^{(34)}$ que la particularité d'un accident extrême réside dans son ampleur, son intensité et l'incapacité à prendre en charge l'ensemble des dommages et des victimes. Force est de constater que dans le cas de Fukushima, l'improbable est devenu réalité: probabilité d'un séisme et d'un tsunami avec celle d'une réaction en chaîne où les trois réacteurs en fonctionnement ont simultanément perdu leur refroidissement en eau et leur alimentation électrique. Le

30 Bernadette de VAnssay, "Quand les sciences humaines éclairent l'analyse des risques», Pouvoirs Locaux. Les Cahiers de la Décentralisation, ${ }^{\circ}$ 56, 1 (2003), p. 56.

31 Francis Eustache, Mémoire et émotions, Paris, Le Pommier (coll. Essais et documents), 2016.

32 Il est d'ailleurs intéressant de noter comment la panique alimentaire, la peur de manger a succédé à la peur de manquer.

33 Valentine Erné-Heintz, «Penser le risque résiduel: l'improbable catastrophe», Risques, Études et Observations, $\mathrm{n}^{\circ} 3$ (2012), www.riseo.cerdacc.uha.fr/. 
caractère apocalyptique des conséquences sur les enjeux positionne l'aléa exceptionnel dans un autre registre. Il est hors-norme ${ }^{(35)}$, hors du cadre. Concernant le risque inondation, l'agglomération strasbourgeoise précise qu'en cas de crue extrême, "trois casernes de pompiers, quatre postes de gendarmerie ou de police, deux mairies et trois établissements hospitaliers, seraient en zone inondée " ${ }^{(36)}$.

Figure 2. Une remise en cause de la loi de causalité ou comment le futur est multiple

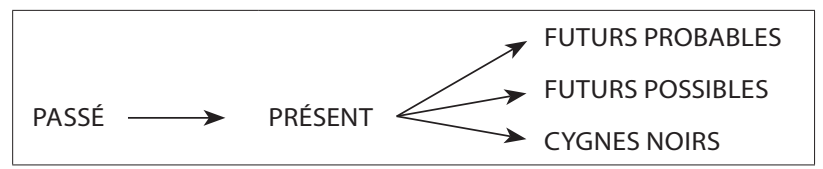

A contrario, les textes législatifs relèvent du registre officiel dans lequel s'organise une gestion du risque bureaucratisée. Ce type de dispositif de sécurité participe à la visibilité du risque et permet aux riverains de vivre avec, de le normaliser. Ils ne s'en préoccupent plus ou peu en raison du caractère administratif et territorialisé de la gestion du risque. Le site industriel est envisagé comme un dossier parmi d'autres - « une affaire courante - et non comme un cas à part ou singulier: l'entreprise ne fait pas problème» ${ }^{(37)}$. Cette planification réduit l'incertitude car elle prévoit un pilotage $a$ priori. Rassurante, cette gestion du risque est prise en charge par des experts, considérés comme des professionnels technico-administratifs ${ }^{(38)}$ : des procédures d'autorisation et des périmètres de sécurité sont fixés, des exercices autour de scénarios d'accident avec le concours de professionnels de la sécurité comme les pompiers sont organisés. Emmanuel Martinais insiste sur cette «fabrique administrative " ${ }^{(39)}$ des plans de prévention du risque industriel par des fonctionnaires du ministère de l'Environnement. Le risque est confiné dans des règles où il s'agit de mettre en conformité des zones ou sites existants avec des textes juridiques (directive Seveso, PPI, PPRT). La rationalité est procédurale puisqu'il s'agit d'un univers marqué par des routines et des règles qui ont pour objectif de mettre en conformité des situations avec des normes préétablies. Ici, le risque n'est plus sanitaire ou environnemental, mais procédural: c'est le défaut d'application d'une règle qui peut être source de conflits ou punissable.

35 Pour de nombreuses raisons: du fait de l'impossibilité à prendre en compte des coûts acceptables, par méconnaissance, par volonté politique ou en lien avec des enjeux économiques...

36 La crue fréquente envisage l'inondation de certaines infrastructures (comme l'aéroport d'Entzheim du fait du débordement de la Bruche, l'interruption de certaines lignes ferroviaires) et de certaines maisons ou quartiers (Montagne-Verte, Plaine des Bouchers, Robertsau). La crue moyenne est également appelée crue centennale: ici, la ville risque d'être significativement impactée (du fait du débordement de l'Ill, de l'Aar et du Rhin). La crue extrême envisage «des débordements généralisés avec des hauteurs d'eau significatives» selon le rapport de présentation de la DREAL Alsace et fragiliserait les capacités à gérer la crise (Dernières Nouvelles d'Alsace du 3 juin 2014, p. 33).

37 Jeanne Снаввац, «Le risque invisible. La non-émergence d’un problème public», Politix, 70/2 (2005), p. 169-195.

38 Comme par exemple les ingénieurs de la DRIRE ou de la DDE, les fonctionnaires de la préfecture parfois spécialisés dans les installations classées.

39 Emmanuel Martinais, «L'écriture des règlements par les fonctionnaires du ministère de l'Écologie. La fabrique administrative du Plan de prévention des risques technologiques», Politix, 90/2 (2010), p. 193-223. 
Le risque extrême n'obéit pas aux mêmes hypothèses qu'un risque ordinaire car le coût d'une catastrophe se révèle immense et la probabilité appartient non pas aux règles des grands nombres (la probabilité n'a pas le statut de fréquences), mais plutôt à celles d'une probabilité bayésienne (c'est-à-dire une information a priori sans expérience statistique de l'événement). De ce fait, il n'entre pas dans le paradigme traditionnel. Cela ne signifie pas que ce dernier l'ignore mais, au contraire, qu'il sous-estime l'éventualité des occurrences... jusqu'à parfois le nier. Et, de ce fait, on en vient à négliger l'aléa exceptionnel ayant un potentiel catastrophique élevé. Il est le plus difficilement envisageable. Il balaye parfois les scénarios les plus élaborés en relayant la prospective en tant que simple utopie. En réalité, la pré-vision (littéralement, "voir avant») révèle le caractère consensuel de la prospective dès lors qu'elle s'en tient à une prise de risque mesurée qui n'implique pas de changement de paradigme, qui ne bouscule pas les normes ou les habitudes décisionnelles. C'est pourquoi le catastrophisme fait référence à une capacité à croire en un possible, généralement celui qui nous paraît impossible:

«Nous étions installés dans le temps des catastrophes. Le monde a vécu l'événement du 11 septembre moins comme l'inscription dans le réel de quelque chose d'insensé, donc d'impossible, que comme l'irruption du possible dans l'impossible. La pire horreur devient désormais possible. Si elle est devenue possible, c'est qu'elle ne l'était pas. Et pourtant, objecte le bon sens, si elle s'est produite, c'est bien qu'elle était possible. [...] C'est bien là la source de notre problème. Car s'il faut prévenir la catastrophe, on a besoin de croire en sa possibilité avant qu'elle ne se produise. Si, inversement, on réussit à la prévenir, sa non-réalisation la maintient dans le domaine de l'impossible, et les efforts de prévention en apparaissent rétrospectivement inutiles ${ }^{(40)}$.

\section{Retour sur la notion de catastrophe}

Comment donner du sens à cet événement redouté dont la présence dans la vie quotidienne reste néanmoins patente: l'usine ou la centrale est installée depuis de nombreuses années, la proximité de la mer offre de nombreuses aménités récréatives, la zone sismique est sous nos pieds, la pression foncière croissante pousse de plus en plus à installer de nouvelles habitations dans des zones sensibles.

\section{Et si le ciel nous tombait sur la tête?}

Une catastrophe collective se caractérise par deux éléments: son imprévisibilité (caractère inédit), l'importance du sinistre et des dommages et la situation de crise qui en découle. De ce fait, la concentration spatiale d'enjeux vulnérables devient un facteur cindynogène majeur. Concernant la tempête Xynthia qui a frappé les littoraux de Vendée et de Charente-Maritime en février 2010, Thierry Feuillet, Étienne Chauveau et Laurent Pourinet constatent que «le caractère exceptionnel de Xynthia relève davantage du nombre de ses victimes (vulnérabilité) que de l'événement météo-marin (aléa) ${ }^{(41)}$. L'indétermination face à l'aléa donne parfois un autre sens à la catastrophe. Citons Venise,

40 J.-P. Dupuy, Pour un catastrophisme éclairé (note 1).

41 Thierry Feuillet, Étienne Chauveau, et Laurent Pourinet. «Xynthia est-elle exceptionnelle? Réflexions sur l'évolution et les temps de retour des tempêtes, des marées de tempête, et des risques de surcotes associés sur la façade atlantique française», Norois, 222/1 (2012), p. 27-44; Valentine Erné-Heintz, «Les élus locaux face au risque inondation ", Environnement et technique, n² 223, janvier-février 2003, p. 48. 
inondée en 2019 par une Acqua Alta, alors que les eaux étaient historiquement protectrices de la population face à d'éventuelles invasions, elles sont devenues une source de danger. Il est important de différencier l'accident ou la catastrophe du risque d'accident ou de catastrophe car la seconde fait référence à la probabilité. Sa représentation relève du virtuel, du potentiel: quand surviendra-t-elle et comment se manifestera-t-elle? Une catastrophe nucléaire est-elle possible en France ${ }^{(42)}$ ? Sommes-nous tous chimiquement contaminés et/ou électro magnétisés ${ }^{(43)}$ ? La catastrophe est une menace qui n'offre que peu de prises car non seulement son anticipation est délicate, mais ses conséquences dépendent d'un nombre important de variables. Ainsi, Fukushima, en 2011, se résume en trois mots: séisme, raz-de-marée et nucléaire. C’est la force de la vague qui a causé le plus de dommages puisque les deux centrales nucléaires installées en bordure de mer ont été submergées. La confiance dans l'un des dispositifs de prévention (le risque sismique) fait négliger les autres types de risque. Par ailleurs, la catastrophe interroge l'opérabilité des diverses ramifications comme les techniques d'assurance ou les fonds d'indemnisation mis en œuvre face à l'ampleur des dommages sanitaires, sociaux ou environnementaux potentiels. Mais chiffrer a-t-il encore un sens? Ainsi, l'IRSN a, en 2013, chiffré le coût d'un accident majeur à plus de 430 milliards d'euros, soit plus de $20 \%$ du PIB français annuel ${ }^{(44)}$. On touche ici aux limites de la capacité à concevoir l'impossible: «on n'arrête pas la mer» et ce, quelle que soit la hauteur de la digue. Ainsi, le vocabulaire utilisé pour caractériser l'ouragan Harvey est éloquent:

«Il s'est installé au lieu de passer son chemin et a déversé des déluges d'eau jamais vus dans l'histoire, noyant au-delà des côtes, jusqu'à la métropole de Houston. On pensait que le danger viendrait du vent, il est venu de l'eau. Un événement imprévisible, puisqu'il ne s'était jamais produit dans ces proportions. [...] Un cygne noir. Un événement improbable aux conséquences d'autant plus désastreuses que l'on ne s'y est pas préparé. [...] L’improbable qui survient et détruit tous les systèmes de pensée » ${ }^{(4)}$.

Assurément, certaines mesures de protection ${ }^{(46)}$ créent l'illusion d'une maîtrise de l'aléa ${ }^{(47)}$. Une catastrophe génère une crise qui, au demeurant, n'est que cyclique

42 L'Institut de radiographie et de sûreté nucléaire (IRSN) indique qu'un accident nucléaire majeur pourrait coûter 420 milliards d'euros, www.irsn.fr/FR/connaissances/Installations_nucleaires/Les-accidentsnucleaires/cout-economique-accident/Pages/2-cout-economique-pour-2-scenarios.aspx\#.W5puJPkzZPY.

43 Élodie Giroux, Martine Hours, «La controverse santé-radiofréquences: la science face à l'incertitude et à la partialité», Environnement, Risques \& Santé, John Libbey Eurotext, 12/3 (2013), p. 222-230, Ffhal-00929707f. Pour ces auteures: «L'exposition aux champs magnétiques est difficilement détectable: ce sont des ondes invisibles et inodores. Par ailleurs, la diffusion des technologies des radiofréquences a été massive et rapide: en quelques années, la téléphonie mobile par exemple a conquis toutes les classes d'âge et tous les milieux. Elle s'est très vite rendue indispensable.»

44 Valentine Erné-Heintz. «Penser le démantèlement d’une centrale nucléaire», Risques, Études et Observations, 2014, p. 127-139, www.riseo.cerdacc.uha.fr/le-demantelement-des-installations-nucleaires-civiles/.

45 Extrait de l'article «Un cygne noir dans le ciel du Texas» paru dans Le Monde du $1^{\text {er }}$ septembre 2017.

46 Les outils de type physique sont divers: création de brises lames insulaires, surélévation de bâtiments, renforcement des écluses, travaux d'enrochements ou de rechargement de plage, réaménagement de berges, reprofilage de cours d'eau, construction d'une digue ou de môles, création de stations de pompage et/ou de champs d'épandage ou encore création d'une porte pour se protéger des vagues de la mer sont autant d'exemples de mesures techniques visant à limiter les effets d'une inondation.

47 Lorsqu’on étudie les conséquences de la tempête Xynthia qui a balayé les côtes atlantiques les derniers jours de février 2010, on observe un raz-de-marée, phénomène appelé «vimer» par les populations 
ou transitoire. Son caractère incontrôlable ou d'une intensité extrême provoque un ébranlement structurel du schéma cognitif. Ainsi, au sujet de Tchernobyl, Ulrich Beck évoque "un choc anthropologique» ${ }^{(48)}$. La catastrophe définit une limite, une rupture. Ainsi en est-il du cyclone Katrina sur la Nouvelle-Orléans (États-Unis) le 29 août 2005: ce cyclone de classe 5 s'accompagna d'une inondation persistante, de catastrophes industrielles en cascade provoquant des pollutions importantes. Les moyens de secours seront limités par l'indisponibilité de certaines infrastructures avec un questionnement sur une éventuelle perte d'une partie de la ville. Au même titre, pour l'ouragan Harvey qui a frappé le Texas en 2017:

«Une hauteur d'eau qui s'est ajoutée à l'onde de tempête soulevée par l'ouragan Harvey, par l'effet des vents très forts poussant les flots et des très basses pressions atmosphériques au cœur de l'ouragan. Des effets combinés qui ont entraîné les eaux à l'intérieur des terres, jusqu'à Houston, amplifiant les dégâts des inondations liées aux précipitations exceptionnelles. [...] Ces défenses sont conçues pour un certain niveau de la mer, et une légère élévation peut suffire pour qu'elles ne soient plus du tout adaptées, ce qui peut entraîner des destructions extrêmement coûteuses » ${ }^{(49)}$.

\section{Plaidoyer pour la prise en compte des scénarios catastrophistes}

Il existe deux approches pour évaluer le risque: une approche probabiliste et une déterministe. L'approche probabiliste place la probabilité d'occurrence au centre de l'analyse. Elle n'est possible que si la réitération des observations (mesures, expériences d'accident) est envisageable. De ce fait, elle se base sur le retour d'expérience en quantifiant la probabilité et les dommages potentiels. L'approche probabiliste fonctionne dans le cadre d'un risque raisonnable ou ordinaire (c'est la pratique des assurances). Elle se justifie dans le cas où des outils de mesure statistiques existent comme la loi des grands nombres (répétition de l'expérience) et la possibilité d'évaluer les dommages potentiels.

«Il est parfois délicat de penser certains types de risques lorsqu’on se trouve dans des situations d'incertitude extrême où la représentation sous forme de risque peut même devenir impossible. C'est le cas lorsque l'on ne peut pas déterminer si le danger existe, ou quelles seraient les conséquences de sa survenue» ${ }^{(50)}$.

En somme, l'approche rationalisée et bureaucratisée des procédures néglige les risques émergents et les signaux d'alerte émanant de sources autres que celles des voies officielles (agences d'expertise) car:

locales. Dans le passé, ce risque, dont les conséquences sont connues, avait justifié la construction d'une digue. Mais elle avait été oubliée et n'avait pas été entretenue. Ainsi, lorsqu'on se concentre sur le détail des dommages, les victimes logent dans des maisons sans étages et sans surélévation; les maisons sont plus proches des digues que les anciennes situées davantage à l'intérieur des terres. Paradoxalement, à l'île de Ré, par exemple, les mesures prises concernent les zones impactées par la tempête. Or, une simple modification de l'orientation des vents déplace les dégâts provoqués par les flots. Pour un détail de la répétition de ce phénomène de vimers, nous invitons le lecteur à consulter les Cahiers de la mémoire de l'île de Ré, n 75, été 2000, réédition 2010.

U. BEск, La société du risque (note 10).

49 Extraits d'un article intitulé «Houston, face au déluge biblique Harvey» paru dans Le Figaro du 29 août 2017, p. 6.

50 Erné-Heintz/Bard, «Un expert précautionneux» (note 13). 
«La suspicion d'effets cocktails et les modes d'action des perturbateurs endocriniens échappent à la toxicologie classique. Évaluer des effets cocktails supposerait si l'on suivait la voie habituelle de multiplier à l'infini les tests toxicologiques. Quant aux perturbateurs endocriniens, ce sont les principes toxicologiques classiques de l'évaluation des risques qui se trouvent pris en défaut» ${ }^{(51)}$.

Figure 3. Le scénario hors-cadre

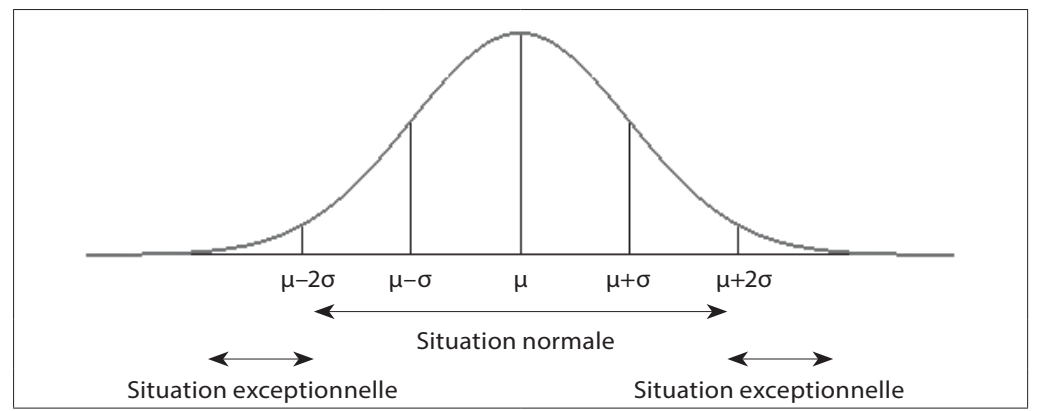

En outre, la question posée se décline également sous l'angle de la définition du caractère extrême de la catastrophe: si la moyenne se déplace, les situations considérées comme extrêmes peuvent devenir «normales» et se repositionner dans la courbe de Gauss (fig. 3). Ainsi, concernant l'évolution du climat, «les ouragans et les inondations représentent près des trois quarts des catastrophes climatiques naturelles, et leur fréquence s'accroît. Si l'on en a compté 200 dans le monde en 1980, il y en a eu plus de 600 cette année» ${ }^{(52)}$. D’ailleurs, "selon la base de données du Centre de recherche sur l'épidémiologie des désastres (CRED), associé à l'université catholique de Louvain, le nombre de catastrophes naturelles survenues dans le monde entre 2005 et 2014 a été multiplié par quatre, par rapport à la décennie 1970-1979» ${ }^{(53)}$.

De son côté, l'approche déterministe élargit les causes et les conséquences envisageables là où le raisonnement probabiliste réduit à néant certaines possibilités. Pour le risque technologique, le plan de prévention des risques technologiques (PPRT) allie les deux approches afin de délimiter des zones avec des potentiels de dangerosité différents tout en prenant en compte la probabilité des scénarios d'accidents possibles ${ }^{(54)}$. C'est pourquoi l'approche déterministe se concentre sur l'évaluation des conséquences sur les enjeux. Dans la gestion de l'urbanisation, la définition de zones à risques (effets létaux, effets irréversibles) dans les plans particuliers d'intervention (PPI) ou PPRT permet de spatialiser l'aléa, de le cartographier pour organiser les secours en cas d'accident. Elle est plus appropriée pour les cas où le risque présente un potentiel

51 Erné-Heintz/Vergnaud, «Au sujet du besoin d'un niveau de preuve robuste pour évaluer le risque» (note 6).

52 Article intitulé «La facture climatique se déchaîne», paru dans Challenges, n 533, 14 septembre 2017, p. 48 et repris par The Economist, Londres, 2017.

53 Le Monde du 9 septembre 2017, p. 2.

54 Valentine Erné-Heintz, «Les enjeux économiques de la planification des risques industriels», in: Jean-François Brilhac, Karine Favro (dir.), Planifier le risque industriel, Paris, Victoires Éditions, 2009, p. 107. 
catastrophique important (accident nucléaire, accident industriel majeur). Elle définit des périmètres ou zones de danger en référence au pire des scénarios. Cependant, cette mise en visibilité souffre parfois d'un défaut d'acceptabilité sociale. Citons les débats autour de l'urbanisation dans des villes comme Lacanau ou encore dans le cadre de la mise en place du PPRT d'une raffinerie:

«Beaucoup d'habitants prennent connaissance à cette occasion des termes de cette réglementation : la possibilité de se faire exproprier, l'obligation de faire des travaux de sécurisation des logements qui concernera beaucoup d'entre eux et auxquels ils devront contribuer financièrement. Cette double information constitue une forme de prise de conscience "forcée" des risques industriels qui agit comme un coup de massue sur les habitants " ${ }^{(55)}$.

En d'autres termes, c'est bien cette incapacité à croire l'inconcevable, à accepter que le pire soit possible qui constitue le principal obstacle à une anticipation de tous les scénarios. Dans l'éthique jonassienne, il s'agit de donner la priorité à la prévention de l'accident le plus grave même si la probabilité qu'il se réalise est nettement plus faible qu'un accident de référence: "La prophétie de malheur est faite pour éviter qu'elle ne se réalise ${ }^{(56)}$. En somme, le catastrophisme invite à développer un cadre prospectif (non probabilisé); il possède une puissante portée anthropique. C’est pourquoi il peut s'interpréter comme des possibles qui invitent à une rétroaction entre le passé et le futur: considérer la catastrophe comme inéluctable peut être la meilleure stratégie pour éviter qu'elle n'advienne réellement. Penser le catastrophisme, c'est éviter que le scénario catastrophique advienne car une fois pensé, il ne pourra pas se produire. Dans la Bible, Noé n'a-t-il pas construit son arche avant qu'il ne pleuve? Le pompier n'entretient-il pas son matériel avant qu'un incendie n'advienne? C'est en ce sens que le catastrophisme se conjugue avec la notion de projet, d'investissement irréversible: ce n'est pas le passé qui conditionne l'avenir mais au contraire le futur qui conditionne le présent à travers la réalisation des projets. Entrevoir une catastrophe comme un futur possible oblige à mettre en place des mesures de gestion préventives; considérer une crue comme un événement envisageable peut susciter des mesures structurelles (déviation et rectification de cours d'eau, endiguements, bassin de rétention) et organisationnelles. C'est justement parce que ce futur n'est pas désiré qu'il doit susciter sa pleine considération dans l'analyse du risque. Ce futur interdit détermine les présents possibles de sorte que le pire ne soit plus à venir. Autrement dit, le catastrophisme inverse la notion de temps: ce n'est plus le passé qui définit le futur, mais le futur proscrit qui influence le présent. Citons l'île de Ré avec la construction d'une porte anti-submersion pour se protéger des vagues de la mer ${ }^{(57)}$ et dont la hauteur a été calculée conjointement en prenant en considération à la fois le scénario d’une très forte tempête et des pires prévisions liées au réchauffement climatique. Le catastrophisme

55 Clara Osadtchy, «Mobilisations et conflits liés à la maîtrise de l'urbanisation autour des industries à risque», Territoire en mouvement - Revue de géographie et aménagement (en ligne), 23-24 (2014), mis en ligne le $1^{\text {er }}$ décembre 2014, consulté le 9 octobre 2018, journals.openedition.org/tem/2618; doi : 10.4000/tem.2618.

56 H. Jonas, Le principe responsabilité (note 7).

57 Sur la commune de La Flotte, une porte anti-submersion de 42 tonnes pour bloquer les entrées d'eau. Exemple extrait du quotidien Sud-Ouest du 21 avril 2016 et qui concerne l'île de Ré: une porte comme pour dire à la mer qu'elle n'est pas la bienvenue. Aujourd'hui, de nombreuses critiques s'élèvent pour dénoncer le «bétonnage» de l'île. 
est consubstantiel à l'aléa extrême ou à l'événement statistiquement aberrant. C'est dans cet esprit que Jean-Pierre Dupuy définit l'avenir probable comme un présent certain qui permet à l'impossible de ne pas se réaliser.

Toutefois, il subsistera toujours une part d'incertitude (du fait de la complexité des systèmes socio-économiques) et qui se traduit par une gestion de crise. En effet, à la différence de la gestion préventive, la gestion de crise intervient pendant ou après la catastrophe et vise explicitement la réduction des dommages : plan d'évacuation, organisation des secours, création de systèmes d'alerte pour les inondations par exemple. Il en est de même pour le risque intrusion depuis les attentats avec le plan particulier de mise en sûreté (PPMS). Une gestion de crise qui prend la forme d'un plan local de gestion de crise (PLGC) se justifie explicitement dans les cas de dysfonctionnements ou de ruptures (défaillance dans les infrastructures, erreurs humaines, etc.).

\section{Conclusion}

Certains risques renouvellent le besoin d'un nouveau cadre cognitif ${ }^{(58)}$. Pour Dupuy $^{(59)}$, le principe de précaution est déjà dépassé. D’ailleurs, face à certaines menaces, préférer utiliser le terme de risque rassure car cela suppose une quantification et une gestion de celui-ci : il serait maîtrisable. Pourtant, la question du catastrophisme s'inscrit dans un autre paradigme qui suppose une approche plus globale qui ne se fonde pas uniquement sur les promesses technologiques. Sherlock Holmes n'avait-il pas pour devise: «quand vous avez éliminé l'impossible, ce qui reste, aussi improbable cela soit-il, doit être la vérité » ? L'ancienne éthique (kantienne) ne tient pas compte du non-humain ou des générations futures; il est alors nécessaire de changer de paradigme, ce qui passe aussi par une révolution épistémologique ${ }^{(60)}$.

C'est pourquoi cette contribution a tenté de montrer qu'une vision catastrophique semblait être, dans certains cas, pertinente et adaptée car elle évite les approches simplificatrices. Elle s'intègre dans un contexte non probabilisable objectivement qui prend en compte d'éventuels points de basculement (tipping point) ou bifurcations, hors des tendances lourdes. Autrement dit, le catastrophisme rappelle simplement que si le futur n'est pas écrit d'avance, il peut néanmoins se préparer.

\section{Résumé}

Le catastrophisme pose la question de l'appréhension des événements extrêmes, qu'ils soient connus et pensés ou inconnus et impensables. Envisager la catastrophe réinsère la prudence dans l'analyse du risque en rappelant que la précaution n'est qu'une traduction de l'éthique de responsabilité chère à Hans Jonas. Il concerne tout autant un risque avéré dont, en général, la gravité est forte et la probabilité très faible qu’un événement

58 Valentine Erné-Heintz, «Que nous apprennent les nouveaux risques? Vers la construction d'un nouveau paradigme ou comment l'effet critique adverse s'impose!», Journal des Accidents et des Catastrophes, avril 2014, www.jac.cerdacc.uha.fr/.

59 J.-P. Dupuy, Pour un catastrophisme éclairé (note 1).

60 Valentine Erné-Heintz, "Croiser les regards pour renouveler l'analyse du risque», Revue d'Allemagne et des pays de langue allemande, 50/2 (2018), p. 345-354. 
dont le scénario reste à construire. Cette contribution revient sur des situations de rupture ou d'aveuglement statistiquement qualifiées d'événements aberrants et génératrices de catastrophes, qui mettent à l'épreuve le cadre cognitif et les procédures classiques d'expertise et de gestion. Sont-elles, pour autant, toujours non prévisibles? Aussi, les propos s'organisent en deux points: premièrement, nous définissons le cadre hors-norme dans lequel cet improbable cygne noir s'épanouit. Deuxièmement, nous caractérisons la notion de catastrophe en insistant sur la nécessité de penser les scénarios catastrophiques pour une meilleure gestion du présent.

\title{
Zusammenfassung
}

Katastrophen werfen die Frage nach extremen Gefährdungen auf, gleichzeitig mit unbekannten, undenkbaren Ereignissen. Denkt man an eine Katastrophe, wird die Vorsicht wieder in die Risikoanalyse einbezogen, wobei man daran erinnert, dass Vorsichtsmaßnahmen nur eine Übersetzung der Ethik der Verantwortung sind, die Hans Jonas am Herzen liegt. Dabei handelt es sich sowohl um ein nachgewiesenes Risiko mit sehr geringer Wahrscheinlichkeit als auch um ein potenzielles Risiko, dessen Szenario noch erstellt werden muss. Dieser Beitrag kehrt zu Situationen von Brüchen oder Blindheit zurück, die statistisch als anormale Ereignisse qualifiziert sind, die herkömmliche Fachkenntnisverfahren auf die Probe stellen. Sind sie immer noch unvorhersehbar? Auch die Ausführungen gliedern sich in zwei Punkte: Erstens definieren wir den außergewöhnlichen Rahmen, in dem dieser unwahrscheinliche schwarze Schwan gedeiht. Zweitens charakterisieren wir den Begriff der Katastrophe, indem wir darauf bestehen, katastrophale Szenarien für ein besseres Management der Gegenwart zu entwickeln.

\begin{abstract}
Doom-mongering raises the question of extreme hazards at the same time as that of unknown, unthinkable events. Thinking about disaster reintroduces caution into risk analysis, reminding us that precaution is only a translation of the ethics of responsibility that are so dear to Hans Jonas. It concerns a proven risk whose probability is very low just as much as it concerns a potential risk whose scenario remains to be constructed. This contribution returns to situations of rupture or blindness that are statistically qualified as aberrant events and which put conventional expertise procedures to the test. Are the risks still unpredictable? To answer the question, this contribution contains two parts. First, we define the extraordinary framework in which an improbable black swan flourishes. Second, we characterize the notion of disaster by insisting on the need to think of catastrophic scenarios to better manage the present.
\end{abstract}

